\title{
The effects of bleeps on medical on-call doctors' workflow and work efficiency
}

\author{
Authors: Fang En Sin,, Gareth Watts* and Edward Kingdon
}

\section{Introduction}

While bleeps are a reliable and rapid method of communication, frequent bleeps cause interruption in patient care and disruption to workflow for the receiver. ${ }^{1,2}$ Furthermore, clinicians sending a bleep can wait an average of 9.35 minutes for a reply, ${ }^{3}$ representing significant inefficiency. Local feedback from junior doctors reflects the above. This project describes the volume of bleeps received by out-of-hours medical doctors within our hospital and the reasons they are sent. Understanding current activity will support planning for effective and efficient communication in future.

\section{Methods}

Switchboard data was inspected to identify the frequency and timing of bleeps sent to doctors on-call covering medical wards at one of two acute sites in over four consecutive weekends. There are five doctors covering medical wards between 9am and 9pm (one specialty registrar (SpR), two general practice specialty registrar / foundation year (FY) 2 and two FY1 grades), one between 2pm and 12am (SpR) and three overnight (one SpR, one core medical trainee (CMT) / FY2 and one FY1). The data presented describes bleeps during a 24-hour period.

On-call junior doctors completed a log over one weekend to capture the indication for each bleep, and whether responding interrupted an existing task.

\section{Results}

A total of 1,632 bleeps were received by eight medical on-call doctors covering the medical wards at the Royal Sussex County Hospital site during 8 consecutive weekend days.

The mean number of bleeps per day per person was 35.9 (range 17-54). The frequency of bleeps to doctors varied by grade. The night foundation year 1 doctor received the most, with 4.5 bleeps an hour.

Assuming 5 minutes spent answering each bleep, an on-call doctor spends an average of 3 hours (range 1.4-4.5) in every 24-hour period answering bleeps. For the bleep sender, this equates to an average of 5.7 hours in a 24 -hour period waiting for a call back.

The pattern of bleeps over time of the day is shown in Fig 1.

The bleep log reveals that $92 \%$ bleeps interrupt existing tasks, with post-take ward rounds ( $42 \%$ ) and clinical reviews (38\%) constituting the majority of these tasks. $49 \%$ of logged bleeps were for routine tasks, defined as tasks that do not need attention

Authors: Brighton and Sussex University Hospitals NHS Trust ${ }^{*} \mathrm{RCP}$ chief registrar

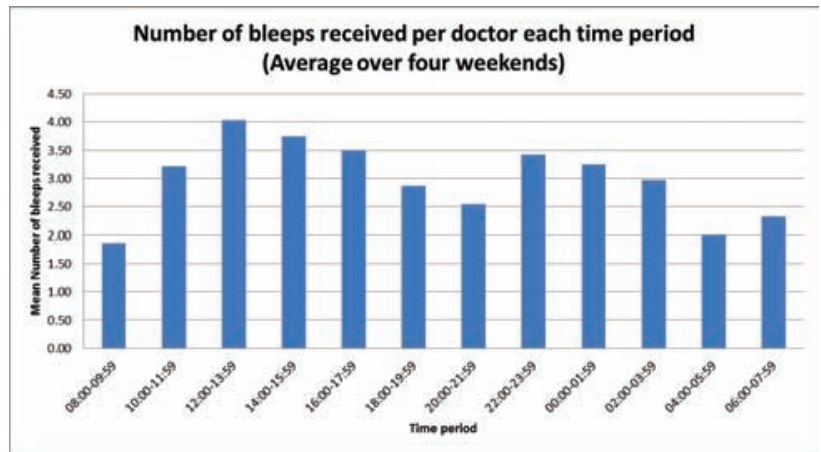

Fig 1. Graph showing time variation in average number of bleeps received by each on-call doctors over a 24-hour period

within 60 minutes. $14 \%$ of bleeps were directed at incorrect doctors and had to be redirected. The return rate of bleep log was incomplete, but our data is consistent with similar published data. ${ }^{2,4}$

\section{Conclusion}

While bleeps are a reliable and effective means of recruiting clinicians to urgent or emergency tasks, using bleeps for non-urgent tasks may compromise the coordinated work of groups of clinicians. Just under half of bleeps received by out-of-hours medical doctors in this study were for non-urgent tasks. Interruption of post-take ward rounds and unscheduled patient reviews reflects a significant opportunity cost for the current system.

We plan to generate updated guidance for non-emergency bleeps, to whom they should be directed and how to minimise ward round interruptions and incorrectly-directed bleeps. We will also supplement the quantitative data with users' perspectives, to help build a strategy to optimise out-of-hours clinical communication.

\section{References}

1 Fargen KM, O'Connor T, Raymond S, Sporrer JM, Friedman WA. An observational study of hospital paging practices and workflow interruption among on-call junior neurological surgery residents. Journal of Graduate Medical Education 2012;4:467-71.

2 Katz MH, Schroeder SA. The sounds of the hospital: paging patterns in three teaching hospitals. N Engl J Med 1988;319:1585-9.

3 Mitchell J, Hayhurst C, Robinson SM. Can a senior house officer's time be used more effectively? Emerg Med J 2004:21:545-7.

4 Blum NJ, Lieu TA. Interrupted care: the effects of paging on pediatric resident activities. Am J Dis Child 1992;146:806-8. 